\title{
Partitions of an Integer into Powers
}

\author{
Matthieu Latapy \\ LIAFA, Université Paris 7, 2 place Jussieu, 75005 Paris. latapy@liafa.jussieu.fr \\ received Febuary 03, 2001, revised April 20, 2001, accepted April 30, 2001.
}

\begin{abstract}
In this paper, we use a simple discrete dynamical model to study partitions of integers into powers of another integer. We extend and generalize some known results about their enumeration and counting, and we give new structural results. In particular, we show that the set of these partitions can be ordered in a natural way which gives the distributive lattice structure to this set. We also give a tree structure which allow efficient and simple enumeration of the partitions of an integer.
\end{abstract}

Keywords: Integer partition, Composition, Lattice, Distributive Lattice, Discrete Dynamical Models, Chip Firing Game

\section{Introduction}

We study here the problem of writing a non-negative integer $n$ as the sum of powers of another positive integer $b$ :

$$
n=p_{0} b^{0}+p_{1} b^{1}+\cdots+p_{k-1} b^{k-1}
$$

with $p_{k-1} \neq 0$ and $p_{i} \in \mathbb{N}$ for all $i$. Following [Rod69], we call the $k$-tuple $\left(p_{0}, p_{1}, \ldots, p_{k-1}\right)$ a $b$-ary partition of $n$. The integers $p_{i}$ are called the parts of the partition and $k$ is the length of the partition. A $b$-ary partition of $n$ can be viewed as a representation of $n$ in the basis $b$, with digits in $\mathbb{N}$. Conversely, given a $k$-tuple $\left(p_{0}, \ldots, p_{k-1}\right)$ and a basis $b$, we will denote by $v_{b}\left(p_{0}, \ldots, p_{k-1}\right)$ the integer $p_{0} b^{0}+p_{1} b^{1}+$ $\cdots+p_{k-1} b^{k-1}$. There is a unique $b$-ary partition such that $p_{i}<b$ for all $i$, and it is the usual (canonical) representation of $n$ in the basis $b$. Here, we consider the problem without any restriction over the parts: $p_{i} \in \mathbb{N}$, which is actually equivalent to say that $p_{i} \in\{0,1, \ldots, n\}$ for all $i$. We will mainly be concerned with the enumeration and counting of the $b$-ary partitions of $n$, for given integers $n$ and $b$.

This natural combinatorial problem has been introduced by Mahler [Mah40], who showed that the $\log$ arithm of the number of $b$-ary partitions of $n$ grows as $\frac{(\log n)^{2}}{2 \log b}$. This asymptotic approximation was later improved by de Bruijn [dB48] and Pennington [Pen53]. Knuth [Knu66] studied the special case where $b=2$. In this case, the function counting the $b$-ary partitions for a given $n$ is called the binary partition function. This function has been widely studied. Euler and Tanturri [Eu150, Tan18a, Tan18b] studied its exact computation and Churchhouse [Chu69, Chu71] studied its congruence properties, while Fröberg [Fro77] gave a final solution to its asymptotical approximation. Later, Rödseth [Rod69] generalized some of these results to $b$-ary partitions for any $b$. Finally, Pfaltz [Pfa95] studied the subcase of the binary partitions of integers which are powers of two. 
We are concerned here with the exact computation of the number of $b$-ary partitions of a given integer $n$, for any $b$. We will use a powerful technique we developped in [LP99] and [LMMP98]: incremental construction of the set of $b$-ary partitions of $n$, infinite extension and coding by an infinite tree. This method gives a deep understanding of the structure of the set of $b$-ary partitions of $n$. We will obtain this way a tree structure which permits the enumeration of all the $b$-ary partitions of $n$ in linear time with respect to their number. We will also order these partitions in a natural way which gives the distributive lattice structure to this set. We recall that a lattice is a partially ordered set such that any two elements $a$ and $b$ have a least upper bound (called supremum of $a$ and $b$ and denoted by $a \vee b$ ) and a greatest lower bound (called infimum of $a$ and $b$ and denoted by $a \wedge b$ ). The element $a \vee b$ is the smallest element among the elements greater than both $a$ and $b$. The element $a \wedge b$ is defined dually. A lattice is distributive if for all $a, b$ and $c:(a \vee b) \wedge(a \vee c)=a \vee(b \wedge c)$ and $(a \wedge b) \vee(a \wedge c)=a \wedge(b \vee c)$. A distributive lattice is a strongly structured set, and many general results, for example efficient coding and algorithms, are known about such sets. For more details, see for example [DP90].

Notice that if we consider $b=1$ and restrict the problem to partitions of length at most $n$, then we obtain the compositions of $n$, i.e. the series of at most $n$ integers, the sum of which equals $n$. Many studies already deal with this special case. In particular, the (infinite) distributive lattice $R_{1}(\infty)$ which we will introduce in Section 4 is isomorphic to the well known Young lattice [Ber71]. Therefore, we will suppose $b>1$ in the following. Notice however that some of the results we present here are already known in this special case (for example the distributive lattice structure), therefore they can be seen as an extension of the existing ones.

\section{The lattice structure}

In this section, we define a simple dynamical model which generates all the $b$-ary partitions of an integer. We will show that the set of $b$-ary partitions, ordered by the reflexive and transitive closure of the successor relation, has the distributive lattice structure.

Let us consider a $b$-ary partition $p=\left(p_{0}, p_{1}, \ldots, p_{k-1}\right)$ of $n$, and let us define the following transition (or rewriting) rule: $p \stackrel{i}{\longrightarrow} q$ if and only if for all $j \notin\{i, i+1\}, q_{j}=p_{j}, p_{i} \geq b, q_{i}=p_{i}-b$ and $q_{i+1}=p_{i+1}+1$ (with the assumption that $p_{k}=0$ ). In other words, if $p_{i}$ is at least equal to $b$ then $q$ is obtained from $p$ by removing $b$ units from $p_{i}$ and adding one unit to $p_{i+1}$. We call this operation firing $i$. The important point is to notice that $q$ is then a $b$-ary partition of $n$. We call $q$ a successor ${ }^{\dagger}$ of $p$, and we denote by $\operatorname{Succ}_{b}(p)$ the set of all the successors of $p$, with respect to the rule. We denote by $R_{b}(n)$ the set of $b$-ary partitions of $n$ reachable from $(n)$ by iterating the evolution rule, ordered by the reflexive and transitive closure of the successor relation. Notice that the successor relation is the covering relation of the order, since it is defined as the transitive and reflexive closure of the successor relation, and one can easily verify that this relation has no reflexive $(x \longrightarrow x)$ and no transitive $(x \rightarrow z$ with $x \rightarrow y$ and $y \longrightarrow z)$ edge. See Figure 1 for some examples.

Given a sequence $f$ of firings, we denote by $|f|_{i}$ the number of firings of $i$ during $f$. Now, consider an element $p$ of $R_{b}(n)$, and two sequences $f$ and $f^{\prime}$ of firings which transform $(n)$ into $p$. Then, $p_{i}=$ $|f|_{i-1}-b \cdot|f|_{i}=\left|f^{\prime}\right|_{i-1}-b \cdot\left|f^{\prime}\right|_{i}$. Suppose that there exists an integer $i$ such that $|f|_{i} \neq\left|f^{\prime}\right|_{i}$, and let $i$ be the smallest such integer. Then, $|f|_{i-1}=\left|f^{\prime}\right|_{i-1}$ and the equality $|f|_{i_{-1}}-b \cdot|f|_{i}=\left|f^{\prime}\right|_{i-1}-b \cdot\left|f^{\prime}\right|_{i}$ is

\footnotetext{
$\dagger$ Notice that the term successor can have many different meanings. We follow here the standard usage in discrete dynamical models, but in order theory the term has another meaning, and one may also consider that a successor of an integer $n$ should be the integer $n+1$, which is not the case here.
} 

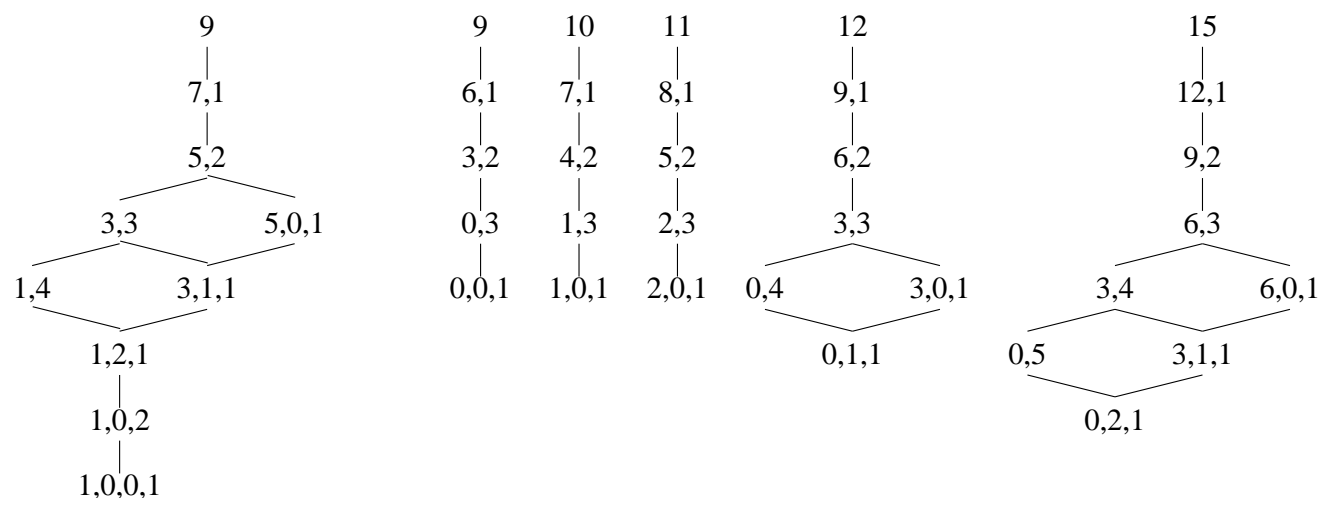

Fig. 1: From left to right, the sets $R_{2}(9), R_{3}(9), R_{3}(10), R_{3}(11), R_{3}(12)$ and $R_{3}(15)$. From Theorem 1, both of these sets is a distributive lattice.

impossible. Therefore, we have $|f|_{i}=\left|f^{\prime}\right|_{i}$ for all $i$. This leads to the definition of the shot vector $s(p)$ : $s(p)_{i}$ is the number of times one have to fire $i$ in order to obtain $p$ from $(n)$. Now we can prove:

Lemma 1 For all $p$ and $q$ in $R_{b}(n), p \leq q$ if and only if for all $i, s(p)_{i} \geq s(q)_{i}$.

Proof : If $p \leq q$, i.e. $p$ is reachable from $q$ then it is clear that for all $i, s(p)_{i} \geq s(q)_{i}$. Conversely, if there exists $i$ such that $s(p)_{i}>s(q)_{i}$, then let $j$ be the smallest such integer. Therefore, $q_{j}>p_{j}+b$ and so $q$ can be fired at $j$. By iterating this process, we finally obtain $p$, and so $p \leq q$.

Theorem 1 For all integers $b$ and $n$, the order $R_{b}(n)$ is a distributive lattice which contains all the $b$-ary partitions of $n$, with the infimum and supremum of any two elements $p$ and $q$ defined by:

$$
s(p \vee q)_{i}=\min \left(s(p)_{i}, s(q)_{i}\right) \text { and } s(p \wedge q)_{i}=\max \left(s(p)_{i}, s(q)_{i}\right) .
$$

Proof : We first show that $R_{b}(n)$ contains all the $b$-ary partitions of $n$. Consider $p$ a $b$-ary partition of $n$. If $p=(n)$, then $p \in R_{b}(n)$, so we suppose that $p \neq(n)$. Therefore, there must be an integer $i>0$ such that $p_{i}>0$. Let us define $q$ such that $q_{j}=p_{j}$ for all $j \notin\{i-1, i\}, q_{i-1}=p_{i-1}+b$ and $q_{i}=p_{i}-1$. It is clear that $q$ is a $b$-ary partition of $n$, and that if $q \in R_{b}(n)$ then $p \in R_{b}(n)$ since $q \stackrel{i-1}{\longrightarrow} p$. It is also obvious that, if we iterate this process, we go back to $(n)$, and so $p \in R_{b}(n)$.

We now prove the formula for the infimum and the supremum. Let $p$ and $q$ be in $R_{b}(n)$, and $r$ such that $s(r)_{i}=\min \left(s(p)_{i}, s(q)_{i}\right)$. From Lemma $1, p$ and $q$ are reachable from $r$. Moreover, if $p$ and $q$ are reachable from $t \in R_{b}(n)$, then, from Lemma $1, r$ is reachable from $t$ since we must have $s(t)_{i} \leq \min \left(s(p)_{i}, s(q)_{i}\right)$ (else one can not transform $t$ into $p$ or $q$ ). Therefore, $r$ is the supremum of $p$ and $q$, as claimed in the theorem. The argument for the infimum is symmetric. Finally, to prove that the lattice is distributive, we only have to check that the formulae satisfy the distributivity laws.

We will now show that the dynamical model defined here can be viewed as a special Chip Firing Game (CFG). A CFG [BLS91, BL92] is defined over a directed multigraph. A configuration of the game is a repartition of a number of chips over the vertices of the graph, and it obeys the following evolution rule: if a vertex $v$ contains as many chips as its outgoing degree $d$, then one can transfer one chip along each of its outgoing edges. In other words, the number of chips at $v$ is decreased by $d$ and, for each vertex $v \neq v$, 
the number of chips at $v$ is increased by the number of edges from $v$ to $v$. This model is very general and has been introduced in various contexts, such as physics, computer science, economics, and others. It is in particular very close to the famous Abelian Sandpile Model [LP00].

It is known that the set of reachable configurations of such a game, ordered with the reflexive and transitive closure of the transition rule, is a Lower Locally Distributive (LLD) lattice (see [Mon90] for a definition and properties), but it is not distributive in general [BL92, LP00, MPV01]. However, if a lattice is LLD and its dual, i.e. the lattice obtained by reversing the order relation, also is LLD, then the lattice is distributive. Therefore, we can give another proof of the fact that $R_{b}(n)$ is a distributive lattice by showing that it is the set of reachable configurations of a CFG, and that its dual too

Given two integers $n$ and $b$, let us consider the following multigraph $G=(V, E)$ defined by: $V=$ $\{0, \ldots, n\}$ and there are $b^{i+1}$ edges from the $i$-th vertex to the $(i+1)$-th, for all $n<i \leq 0$. Now, let us consider the CFG $C$ defined over $G$ by the initial configuration where the vertex 0 contains $n$ chips, the other ones being empty. Now, given a configuration $c$ of the CFG, where $c_{i}$ denotes the number of chips in the vertex number $i$, let us denote by ${ }^{-} c$ the vector such that ${ }_{i} c=\frac{c_{i}}{b^{i}}$. Then, if the CFG is in the configuration $c$, an application of the rule to the vertex number $i$ gives the configuration $c^{\prime}$ such that $c_{i}^{\prime}=c_{i}-b^{i+1}, c_{i+1}^{\prime}=c_{i+1}+b^{i+1}$ and $c_{j}^{\prime}=c_{j}$ for all $j \notin\{i, i+1\}$. Notice that this means exactly that $\bar{c}_{\bar{\epsilon}}$ is decreased by $b$ and that ${ }^{-}{ }_{+1}$ is increased by 1 , therefore an application of the CFG rule corresponds exactly to an application of the evolution rule we defined above, and so the set of reachable configurations of the CFG is isomorphic to $R_{b}(n)$. This leads to the fact that $R_{b}(n)$ is a LLD lattice.

Conversely, let $G^{\prime}$ be the multigraph obtained from $G$ by reversing each edge, and let us consider the CFG $C^{\prime}$ over $G^{\prime}$ such that the initial configuration of $C^{\prime}$ is the final configuration of $C$. Then it is clear that the set of reachable configurations of $C^{\prime}$ is nothing but the dual of the one of $C$, therefore it is isomorphic to the dual of $R_{b}(n)$. This leads to the fact that the dual of $R_{b}(n)$ is a LLD lattice, which allows us to conclude that $R_{b}(n)$ is a distributive lattice.

\section{From $R_{b}(n)$ to $R_{b}(n+1)$}

In this section, we give a method to construct the transitive reduction (i.e. the successor relation) of $R_{b}(n+1)$ from the one of $R_{b}(n)$. In the following, we will simply call this the construction of $R_{b}(n+1)$ from $R_{b}(n)$. This will show the self-similarity of these sets, and give a new way, purely structural, to obtain a recursive formula for $\left|R_{b}(n)\right|$, which is previously known from [Rod69] (the special case where $b=2$ is due to Euler [Eul50]). This construction will also show the special role played by certain $b$-ary partitions, which will be widely used in the rest of the paper. Therefore, we introduce a few notations about them. We denote by $P_{i}(b, n)$ the set of the partitions $p$ in $R_{b}(n)$ such that $p_{0}=p_{1}=\cdots=p_{i-1}=b-1$. Notice that for all $i$ we have $P_{i}(b, n) \subseteq P_{i+1}(b, n)$ and that $P_{0}(b, n)=R_{b}(n)$. If $p=\left(p_{0}, \ldots, p_{k-1}\right)$ is in $P_{i}(b, n)$, we denote by $p^{\hookrightarrow_{i}}$ the $k$-uple $\left(0, \ldots, 0, p_{i}+1, p_{i+1}, \ldots, p_{k-1}\right)$. In other words, $p^{\hookrightarrow}{ }_{i}$ is obtained from $p$ by switching all the $i$ first components of $p$ from $b-1$ to 0 and adding one unit to its $i$-th componend ${ }^{\S}$. Notice that the $k$-uple $p^{\hookrightarrow}$, which is simply obtained from $p$ by adding one unit to its first component, is always a $b$-ary partition of $n+1$. If $S$ is a subset of $P_{i}(b, n)$, we denote by $S^{\hookrightarrow} i$ the set $\left\{p^{\hookrightarrow_{i}} \mid p \in S\right\}$.

Notice that, if $p \stackrel{i}{\rightarrow} q$ in $R_{b}(n)$, then $p \hookrightarrow_{0} \stackrel{i}{\rightarrow} q q^{\hookrightarrow}$ in $R_{b}(n+1)$. This remark makes it possible to construct $R_{b}(n+1)$ from $R_{b}(n)$ : the construction procedure starts with the lattice $R_{b}(n) \hookrightarrow_{0}$ given by its

\footnotetext{
$\$$ This idea is due to Clémence Magnien, who introduced this new way to prove that a set is a distributive lattice using two Chip Firing Games.

$\S$ This operator is known in numeration studies as an odometer. See [GLT95] for more precisions.
} 
diagram. Then, we look for those elements in $R_{b}(n) \hookrightarrow_{0}$ that have a successor out of $R_{b}(n) \hookrightarrow_{0}$. The set of these elements will be denoted by $I_{0}$, with $I_{0} \subseteq R_{b}(n) \hookrightarrow^{0}$. At this point, we add all the missing successors of the elements of $I_{0}$. The set of these new elements will be denoted by $C_{0}$. Now, we look for the elements in $C_{0}$ that have a successor out of the constructed set. The set of these elements is denoted by $I_{1}$. More generally, at the $i$-th step of the procedure we look for the elements in $C_{i-1}$ with missing successors and call $I_{i}$ the set of these elements. We add the new successors of the elements of $I_{i}$ and call the set of these new elements $C_{i}$. At each step, when we add a new element, we also add its covering relations. Since $R_{b}(n+1)$ is a finite set, this procedure terminates. At the end, we obtain the whole set $R_{b}(n+1)$. In the rest of this section, we study more precisely this construction process.

Lemma 2 Let $p$ be a $b$-ary partition in $P_{i}(b, n)$. If $p_{i} \neq b-1$ then $\operatorname{Succ}_{b}\left(p^{\hookrightarrow_{i}}\right)=\operatorname{Succ}_{b}(p)^{\hookrightarrow_{i}}$. Else, $\operatorname{Succ}_{b}\left(p^{\hookrightarrow}\right)=\operatorname{Succ}_{b}(p)^{\hookrightarrow} \cup\left\{p^{\hookrightarrow i+1}\right\}$.

Proof : If a transition $p \stackrel{j}{\rightarrow} q$ is possible, then $p^{\hookrightarrow_{i}} \stackrel{j}{\longrightarrow} q^{\hookrightarrow_{i}}$ is obviously possible. Moreover, an additional transition is possible from $p \hookrightarrow_{i}$ if and only if $p_{i}=b-1$. In this case, $p^{\hookrightarrow_{i}} \stackrel{i}{\rightarrow} p^{\hookrightarrow} \hookrightarrow_{i+1}$.

Lemma 3 For all integer $b, n$ and $i$, we define the function $r_{i}: P_{i}(b, n) \rightarrow R_{b}\left(\frac{n+1}{b^{i}}-1\right)$ by: $r_{i}(p)$ is obtained from $p \in P_{i}(b, n)$ by removing its $i$ first components (which are equal to $\left.b-1\right)$. Then, $r_{i}$ is a bijection.

Proof : Let us consider $p$ in $P_{i}(b, n): p=\left(b-1, b-1, \ldots, b-1, p_{i}, \ldots, p_{k}\right)$. Then, it is clear that $r_{i}(p)=$ $\left(p_{i}, \ldots, p_{k}\right)$ is in $R_{b}\left(\frac{n-(b-1)-(b-1) b-\cdots-(b-1) b^{i-1}}{b^{i}}\right)=R_{b}\left(\frac{n+1-b^{i}}{b^{i}}\right)=R_{b}\left(\frac{n+1}{b^{i+1}}-1\right)$. Conversely, if we consider $p$ in $R_{b}\left(\frac{n+1}{b^{i}}-1\right)$, then $r_{i}^{-1}(p)=\left(b-1, b-1, \ldots, b-1, p_{0}, p_{1}, \ldots, p_{k}\right)$ is a $b$-ary partition of $m=(b-$ $1)+(b-1) b+\cdots+(b-1) b^{i-1}+\frac{n+1-b^{i}}{b^{i}}$, which is nothing but $n$. Therefore, $r_{i}^{-1}(p)$ is in $R_{b}(n)$.

Lemma 4 For all integer $b, n$ and $i$, we have $I_{i}=P_{i+1}(b, n)^{\hookrightarrow_{i}}$ and $C_{i}=P_{i+1}(b, n) \hookrightarrow_{i+1}$.

Proof : By induction over $i$. For $i=0$, it is clear from Lemma 2 that the set of elements in $R_{b}(n) \hookrightarrow_{0}$ with a missing successor, namely $I_{0}$, is exactly $P_{1}(b, n) \hookrightarrow_{0}$. Moreover, the set of these missing successors, namely $C_{0}$, is clearly $P_{1}(b, n)^{\hookrightarrow}$. Now, let us suppose that the claim is proved for $i$ and let us prove it for $i+1$. The set $I_{i+1}$ is the set of elements in $C_{i}$ with one missing successor. By induction hypothesis, we have $C_{i}=P_{i+1}(b, n)^{\hookrightarrow_{i+1}}$ and so, from Lemma $2, I_{i+1}=P_{i+2}(b, n)^{\hookrightarrow_{i+1}}$. Then, by application of the evolution rule, it is clear that the set $C_{i+1}$ of the missing successor is $P_{i+2}(b, n) \hookrightarrow_{i+2}$, which proves the claim.

Theorem 2 For any positive integer $b$ and $n$, we have:

$$
\begin{gathered}
R_{b}(n)=\bigsqcup_{i \geq 0} r_{i}^{-1}\left(R_{b}\left(\frac{n}{b^{i}}-1\right)\right)^{\hookrightarrow_{i}} \\
\left|R_{b}(n)\right|=\sum_{i=0}^{\lfloor n / b\rfloor}\left|R_{b}\left(\frac{i}{b}\right)\right|
\end{gathered}
$$

where $\sqcup$ denotes the disjoint union, where $R_{b}(n)$ is taken as $\emptyset$ when $n$ is not a positive integer, and with $R_{b}(0)=\{0\}$. 
Proof : From the construction procedure described above, we have $R_{b}(n)=R_{b}(n-1) \hookrightarrow_{0} \sqcup \bigsqcup_{i>0} C_{i}$. From Lemma 4, we obtain $R_{b}(n)=R_{b}(n-1)^{\hookrightarrow} \hookrightarrow_{0} \sqcup \bigsqcup_{i>0} P_{i+1}(b, n)^{\hookrightarrow_{i+1}}$. Moreover, since $R_{b}(n-1)^{\hookrightarrow} \hookrightarrow^{\iota_{0}}$ is nothing but $P_{0}(b, n)^{\hookrightarrow_{0}}$, this is equivalent to $R_{b}(n)=\bigsqcup_{i \geq 0} P_{i}(b, n)^{\hookrightarrow_{i}}$. Finally, from Lemma 3 , we obtain the announced formula.

From this formula, we have $R_{b}\left(\frac{n}{b}\right)=\bigsqcup_{i \geq 0} r^{-1}\left(R_{b}\left(\frac{n}{b^{i+1}}-1\right)^{\hookrightarrow_{i}}\right)$. Therefore, $\left|R_{b}(n)\right|=\sum_{i \geq 0} \mid R_{b}\left(\frac{n}{b^{i}}-\right.$ 1) $|=| R_{b}(n-1)\left|+\sum_{i \geq 0}\right| R_{b}\left(\frac{n}{b^{i+1}}-1\right)|=| R_{b}(n-1)|+| R_{b}\left(\frac{n}{b}\right) \mid$. We obtain the claim by iterating this last formula.

The first formula given in this theorem can be used to compute the sets $R_{b}(n)$ efficiently since it only involves disjoint unions. We will give in Section 5 another method to compute $R_{b}(n)$ which is much simplier, as it gives $R_{b}(n)$ a tree structure. However, the formula is interesting since it points out the self-similar structure of the set (see Figure 4).

The second formula is previouly known from [Rod69], and from [Eu150] in the special case where $b=2$. Notice that this does not give a way to compute $\left|R_{b}(n)\right|$ in linear time with respect to $n$, which is an unsolved problem in the general case, but it gives a very simple way to compute recursively $\left|R_{b}(n)\right|$.

\section{Infinite extension}

$R_{b}(n)$ is the lattice of the $b$-ary partitions of $n$ reachable from $(n)$ by iteration of the evolution rule. We now define $R_{b}(\infty)$ as the set of all $b$-ary partitions reachable from $(\infty)$. The order on $R_{b}(\infty)$ is the reflexive and transitive closure of the successor relation. For $b=2$, the first $b$-ary partitions in $R_{b}(\infty)$ are given in Figure 2 along with their covering relation (the first component, which is always infinity, is not represented on this diagram). Notice that it is still possible to define the shot vector $s(p)$ of an element $p$ of $R_{b}(\infty)$ by: $s(p)_{i}$ is the number of times one has to fire $i$ in order to obtain $p$ from $(\infty)$.

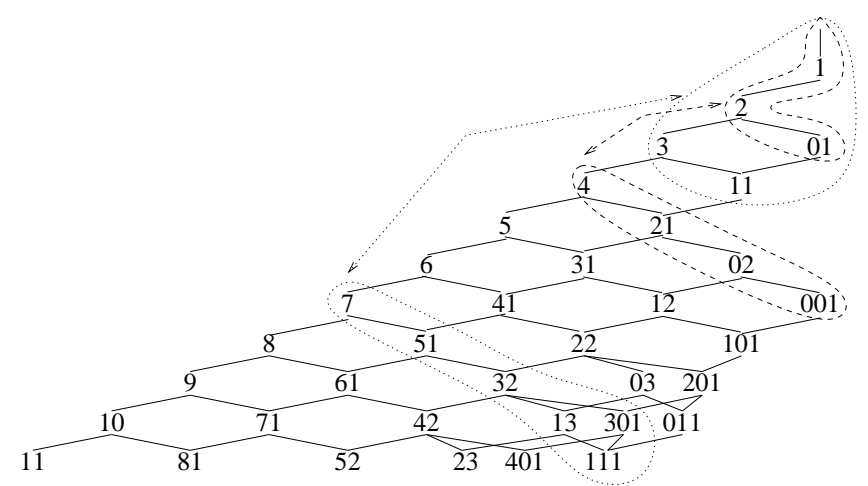

Fig. 2: The first $b$-ary partitions obtained in $R_{b}(\infty)$ when $b=2$. Two parts isomorphic to $R_{2}(4)$ are distinguished, as well as two parts isomorphic to $R_{2}(7)$.

Theorem 3 The set $R_{b}(\infty)$ is a distributive lattice with:

$$
s(p \vee q)_{i}=\min \left(s(p)_{i}, s(q)_{i}\right) \text { and } s(p \wedge q)_{i}=\max \left(s(p)_{i}, s(q)_{i}\right)
$$


for all $p$ and $q$ in $R_{b}(\infty)$. Moreover, for all $n$ the functions

$$
\pi: s=\left(s_{1}, s_{2}, \cdots, s_{k}\right) \longrightarrow \pi(s)=\left(\infty, s_{2}, \ldots, s_{k}\right)
$$

and

$$
\tau: s=\left(s_{1}, s_{2}, \cdots, s_{k}\right) \longrightarrow \tau(s)=\left(\infty, s_{1}, s_{2}, \ldots, s_{k}\right)
$$

are lattice embeddings of $R_{b}(n)$ into $R_{b}(\infty)$.

Proof : The proof for the distributive lattice structure and for the formulae of the infimum and supremum is very similar to the proof of Theorem 1 . Therefore, it is left to the reader.

Given $p$ and $q$ in $R_{b}(n)$, we now prove that $\pi(p) \vee \pi(q)=\pi(p \vee q)$. From Theorem 1 , we have $s(p \vee$ $q)_{i}=\min \left(s(p)_{i}, s(q)_{i}\right)$. Moreover, it is clear that $s(\pi(x))_{i}=s(x)_{i}$ for all $x$ in $R_{b}(n)$. Therefore, $s(\pi(p \vee$ $\left.q))_{i}=\min \left(s(\pi(p))_{i}, s(\pi(q))_{i}\right)\right)$, which shows that $\pi$ preserves the supremum. The proof of $\pi(p) \wedge \pi(q)=$ $\pi(p \wedge q)$ is symmetric. Therefore, $\pi$ is a lattice embedding.

The proof for $\tau$ is very similar when one has noticed that the shot vector of $\tau(s)$ is obtained from the one of $s$ by adding a new first component equal to $n$.

With similar arguments, one can easily show that $\pi\left(R_{b}(n)\right)$ is a sublattice of $\pi\left(R_{b}(n+1)\right)$, and so we have an infinite chain of distributive lattices:

$$
\pi\left(R_{b}(0)\right) \leq \pi\left(R_{b}(1)\right) \leq \cdots \leq \pi\left(R_{b}(n)\right) \leq \pi\left(R_{b}(n+1)\right) \leq \cdots \leq R_{b}(\infty),
$$

where $\leq$ denotes the sublattice relation. Moreover, one can use the self-similarity estalished here to construct filters of $R_{b}(\infty)$ (a filter of a poset is an upper closed part of the poset). Indeed, if one defines $R_{b}(\leq n)$ as the sub-order of $R_{b}(\infty)$ over $\cup_{i \leq n} R_{b}(i)$, then one can construct efficiently $R_{b}(\leq n+1)$ from $R_{b}(\leq n)$ by extracting from $R_{b}(\leq n)$ a part isomorphic to $R_{b}(n+1)$ and pasting it to $R_{b}(\leq n)$. See Figures 2 and 4 .

Notice that, for all integer $b, R_{b}(\infty)$ contains exactly all the finite sequences of integers, since any such sequence can be viewed as a $b$-ary partition of an integer $n$. Therefore, we provide infinitely many ways to give the set of finite sequences of integers the distributive lattice structure.

\section{Infinite tree}

As shown in our construction of $R_{b}(n+1)$ from $R_{b}(n)$, each $b$-ary partition $p$ in $R_{b}(n+1)$ is obtained from another one $p^{\prime} \in R_{b}(n)$ by application of the $\hookrightarrow$ operator: $p=p^{\prime \hookrightarrow}{ }_{i}$ with $i$ an integer between 0 and $l\left(p^{\prime}\right)$, where $l\left(p^{\prime}\right)$ denotes the number of $b-1$ at the beginning of $p^{\prime}$. Thus, we can define an infinite tree $T_{b}(\infty)$ whose nodes are the elements of $\bigsqcup_{n \geq 0} R_{b}(n)$ and in which the fatherhood relation is defined by:

$$
q \text { is the }(i+1) \text {-th son of } p \text { if and only if } q=p^{\hookrightarrow_{i}} \text { for some } i, 0 \leq i \leq l(p) \text {. }
$$

The root of this tree is $(0)$ and each node $p$ of $T_{b}(\infty)$ has $l(p)+1$ sons. The first levels of $T_{b}(\infty)$ when $b=2$ are shown in Figure 3 (we call the set of elements of depth $n$ the "level $n$ " of the tree).

Proposition 1 The level $n$ of $T_{b}(\infty)$ contains exactly the elements of $R_{b}(n)$.

Proof : Straightforward from the construction of $R_{b}(n+1)$ from $R_{b}(n)$ given above and the definition of the tree.

If we define $\overline{R_{b}(n)}$ as $\left\{\left(s_{2}, \ldots, s_{k}\right) \mid\left(s_{1}, s_{2}, \ldots, s_{k}\right) \in R_{b}(n)\right\}$, then: 


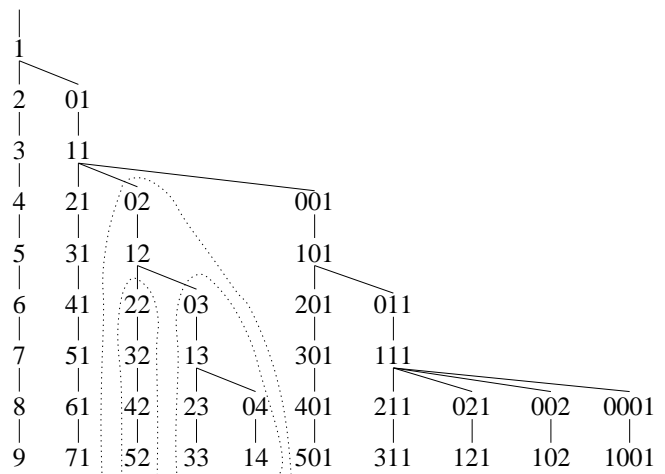

Fig. 3: The first levels of $T_{b}(\infty)$ when $b=2$. We distinguished some special subtrees, which will play an important role in the following.

Proposition 2 For all integer $n$, the elements of $\overline{R_{b}(n)}$ are exactly the elements of the $\left\lfloor\frac{n}{b}\right\rfloor$ first levels of $T_{b}(\infty)$.

Proof : Let us first prove that the elements of $R_{b}(n)$ are the nodes of a subtree of $T_{b}(\infty)$ that contains its root. This is obviously true for $n=0$. The general case follows by induction, since by construction the elements of $\overline{R_{b}(n+1)} \backslash \overline{R_{b}(n)}$ are sons of elements of $\overline{R_{b}(n)}$.

Now, let us consider an element $e$ of the $l$-th level of $T_{b}(\infty)$. If there is a $b$-ary partition $p$ of $n$ such that $\bar{p}=e$, then clearly $p_{i}=e_{i-1}$ for all $i>0$ and $p_{0}=n-b \cdot l$. Therefore, if $e$ is in $\overline{R_{b}(n)}$ then all the elements of the $l$-th level are in $\overline{R_{b}(n)}$, and this is clearly the case exactly when $0 \leq l<\left\lfloor\frac{n}{b}\right\rfloor$. This ends the proof.

Notice that this proposition gives a simple way to enumerate the elements of $R_{b}(n)$ for any $n$ in linear time with respect to their number, since it gives this set a tree structure. Algorithm 1 acheives this.

We will now show that $T_{b}(\infty)$ can be described recursively, which allows us to give a new recursive formula for $\left|R_{b}(n)\right|$. In order to do this, we will use a series known as the $b$-ary carry sequence [Slo73]: $c_{b}(n)=k$ if $b^{k}$ divides $n$ but $b^{k+1}$ does not. Notice that this function is defined only for $n>0$ (or one can consider that $c_{b}(0)=\infty$ ). These series appear in many contexts, and have many equivalent definitions $\mathrm{II}$. Here, we will mainly use the fact that the first $n$ such that $c_{b}(n)=k$ is $n=b^{k}$, and the fact that $c_{b}(n)$ is nothing but the number of components equal to $b-1$ at the begining of the canonical representation of $n-1$ in the basis $b$.

Definition 1 Let $p \in T_{b}(\infty)$. Let us consider the rightmost branch of $T_{b}(\infty)$ rooted at $p$ ( $p$ is considered as the first node of the branch). We say that $p$ is the root of a $X_{b, k}$ subtree (of $\left.T_{b}(\infty)\right)$ if this rightmost branch is as follows: for $i \leq b^{k-1}$, the $i$-th node on the branch has $j=c_{b}(i)+1$ sons, and the l-th $(1 \leq l<j)$ of these sons is the root of a $X_{b, l}$ subtree. Moreover, the $\left(b^{k-1}+1\right)$-th node of the branch is itself the root of $a X_{b, k}$ subtree.

For example, we show in Figure 3 a $X_{2,2}$ subtree of $T_{2}(\infty)$, composed of a $X_{2,1}$ subtree and another $X_{2,2}$ subtree. Notice that a $X_{b, 1}$ subtree is simply a chain.

\footnotetext{
$\mathbb{I}$ For example, if one defines the series $C_{b, 0}=0$ and $C_{b, i}=C_{b, i-1}, \overbrace{i, C_{b, i-1}}^{b-1 \text { times }}$, then $c_{b}(i)$ is nothing but the $i$-th integer of the series $C_{b, i}$. The ten first values for $c_{2}(i)$ are $0,1,0,2,0,1,0,3,0,1$ and the ten first ones for $c_{3}(i)$ are $0,0,1,0,0,1,0,0,2,0$.
} 


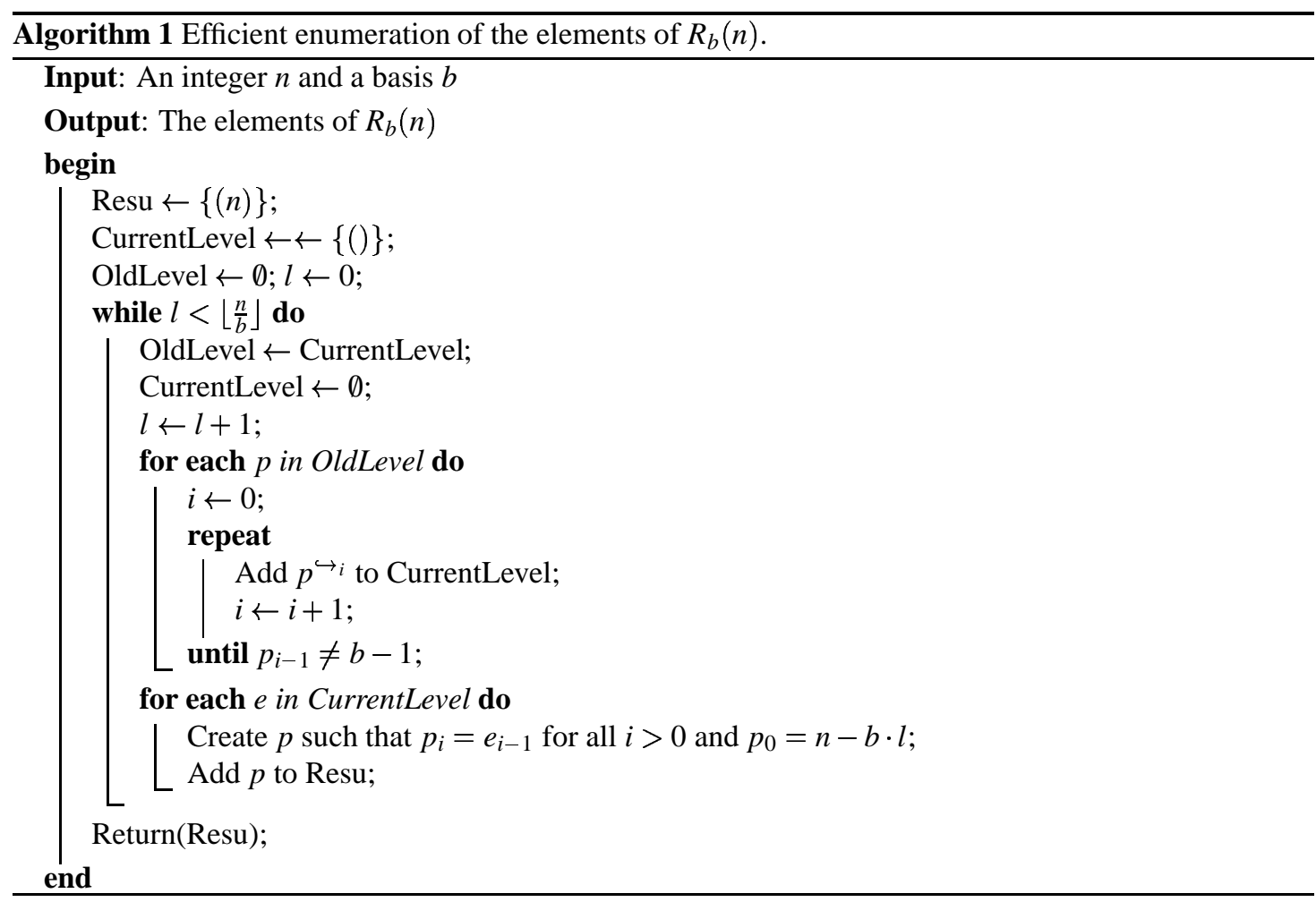


Proposition 3 Let $p=\left(0,0, \ldots, 0, p_{k}, \ldots\right)$ in $T_{b}(\infty)$ with $p_{k}>b-1$. Then, $p$ is the root of $a X_{b, k+1}$ subtree of $T_{b}(\infty)$.

Proof : The proof is by induction over $k$ and the depth of $p$. Let us consider the rightmost branch rooted at $p$. Since, for all $q$ in $T_{b}(\infty)$, the rightmost son of $q$ is $q^{\hookrightarrow} \hookrightarrow_{i}$ with $i$ the number of $b-1$ at the beginning of $q$, it is clear that the $j$-th node of this branch for $j \leq b^{k}$ is $q=\left(q_{0}, \ldots, q_{k-1}, p_{k}, \ldots\right)$ where $\left(q_{0}, \ldots, q_{k-1}\right)$ is the canonical representation of $j-1$ in the basis $b$. Therefore, $q$ begins with $c_{b}(j)$ components equal to $b-1$, and so, for $l=1, \ldots, c_{b}(j)$, the $l$-th son of $q$ starts with $l-1$ zeroes followed by a component equal to $b>b-1$. By induction hypothesis, we then have that the sons of $q$ are the roots of $X_{b, l}$ subtrees. Moreover, the $\left(b^{k}+1\right)$-th node on the rightmost branch begins with exactly $k$ zeroes followed by a component greater than $b-1$, and so it is the root of a $X_{b, k+1}$ subtree by induction hypothesis.

Theorem 4 The infinite tree $T_{b}(\infty)$ is a $X_{b, \infty}$ tree: it is a chain (its rightmost branch) such that its $i$-th node has $c_{b}(i)$ sons and the $j$-th of these sons, $1 \leq j \leq c_{b}(i)$, is the root of a $X_{b, j}$ subtree. Moreover, the $i$-th node of the chain is the canonical representation of $i-1$ in the basis $b$.

Proof : Since the rightmost son of $p \in T_{b}(\infty)$ is $p^{\hookrightarrow}$, where $i$ is the number of $b-1$ at the beginning of $p$, and since the root of $T_{b}(\infty)$ is nothing but the canonical representation of 0 , it is clear by induction that the $i$-th node of the rightmost branch of $T_{b}(\infty)$ is the canonical representation of $i-1$ in the basis $b$. Then, the theorem follows from Proposition 3.

We now have a recursive description of $T_{b}(\infty)$, which allows us to give recursive formula for the cardinal of some special sets. Let us denote by $\pi_{b}(l, k)$ the number of paths of length exactly $l$ starting from the root of a $X_{b, k}$ subtree of $T_{b}(\infty)$. We have:

\section{Theorem 5}

$$
\pi_{b}(l, k)= \begin{cases}1 & \text { if } 0 \leq l<b \\ 1+\sum_{i=1}^{l} \sum_{j=1}^{c_{b}(i)} \pi_{b}(l-i, j) & \text { if } b \leq l \leq b^{k-1} \\ \pi_{b}\left(l-b^{k-1}, k\right)+\sum_{i=1}^{b^{k-1}} \sum_{j=1}^{c_{b}(i)} \pi_{b}(l-i, j) & \text { otherwise }\left(l>b^{k-1}\right)\end{cases}
$$

Moreover, $\left|R_{b}(n)\right|=\pi_{b}(n, n)$ and the number of $b$-ary partitions of $n$ into exactly l parts is $\pi_{b}(n-(b-$ $\left.1)^{l}, l\right)$.

Proof : The formula for $\pi_{b}(l, k)$ is directly deduced from the definition of the $X_{b, k}$ subtrees. The other formulae derive from Theorem 4 and from the fact that all the $b$-ary partitions of length $l$ are in a $X_{b, l}$ subtree of $T_{b}(\infty)$ which is rooted at the $(b-1)^{l}$-th node of the righmost branch of $T_{b}(\infty)$.

\section{Perspectives}

The results presented in this paper mainly point out the strong self-similarity and the structure of the sets $R_{b}(n)$. As already noticed, it is an open question to compute the cardinal of $R_{b}(n)$ in linear time with respect to $n$, and one may expect to obtain a solution using these results.

Another interesting direction is to investigate how one can extend the dynamics we study. A first idea is to consider non-integer basis, in particular complex basis or Fibonnacci basis. For example, if we consider the complex basis $b=i-1$ then we can obtain all the ways to write an integer $n$ as the sum of powers of $b$ by iterating the following evolution rule from $(n): q$ is a successor of $p$ if $p-q=$ 
$(0, \ldots, 0,2,0,-1,-1,0 \ldots, 0)$. In other words, we can decrease by two the $j$-th component of $p$ and increase by one its $(j+2)$-th and its $(j+3)$-th components for some integer $j$. This gives to the set of representations of $n$ in the complex basis $b=i-1$ the lattice structure, since this can be encoded by a Chip Firing Game [LP00] (notice however that in this case the lattice is no longer distributive). Another interesting case is when $b=1$. As already noticed, we obtain the Young lattice, or equivalently the lattice of the compositions of $n$.

\section{Acknowledgments}

I thank Christiane Frougny and Clémence Magnien for many useful comments on preliminary versions, which deeply improved the manuscript.

\section{References}

[Ber71] Claude Berge. Principles of Combinatorics, volume 72 of Mathematics in science and engineering. Academic Press, 1971.

[BL92] A. Bjorner and L. Lovász. Chip-firing games on directed graphs. J. Algebraic Combinatorics, $1: 305-328,1992$.

[BLS91] A. Bjorner, L. Lovász, and W. Shor. Chip-firing games on graphs. E.J. Combinatorics, 12:283-291, 1991.

[Chu69] R.F. Churchhouse. Congruence properties of the binary partition function. Proc. Camb. Phil. Soc, 66:371-375, 1969.

[Chu71] R.F. Churchhouse. Binary partitions. In A.O.L. Atkin and B.J. Birch, editors, Computers in Number Theory, pages 397-400. Academic Press, 1971.

[dB48] N.G. de Bruijn. Nederl. Akad. Wetensch. Proc., 51:659-669, 1948.

[DP90] B.A. Davey and H.A. Priestley. Introduction to Lattices and Orders. Cambridge university press, 1990.

[Eul50] L. Euler. Novi Comm. Petrop., III, 1750.

[Fro77] C.-E. Froberg. Accurate estimation of the number of binary partitions. BIT, 17:386-391, 1977.

[GLT95] P.J. Grabner, P. Liarded, and R.F. Tichy. Odometers and systems of numeration. Acta Arithmetica, LXX.2:103-123, 1995.

[Knu66] D.E. Knuth. An almost linear recurrence. Fib. Quart., 4:117-128, 1966.

[LMMP98] M. Latapy, R. Mantaci, M. Morvan, and H.D. Phan. Structure of some sand piles model. 1998. To appear in Theoretical Computer Science, preprint available at http://www.liafa.jussieu.fr/ latapy/. 
[LP99] M. Latapy and H.D. Phan. The lattice of integer partitions and its infinite extension. 1999. To appear in DMTCS, special issue, proceedings of ORDAL'99. Preprint available at http://www.liafa.jussieu.fr/ latapy/.

[LP00] M. Latapy and H.D. Phan. The lattice structure of chip firing games. 2000. To appear in Physica D. Preprint available at http://www.liafa.jussieu.fr/ latapy/.

[Mah40] Kurt Mahler. On a special functional equation. J. London Math. Soc, 15:115-123, 1940.

[Mon90] B. Monjardet. The consequences of Dilworth's work on lattices with unique irreducible decompositions. In K.P.Bogart, R.Freese, and J.Kung, editors, The Dilworth theorems. Selected papers of Robert p. Dilworth, pages 192-201. Birkhauser, Boston, 1990.

[MPV01] C. Magnien, H.D. Phan, and L. Vuillon. An extension of the chip firing game. 2001. preprint.

[Pen53] W.B. Pennington. On Mahler's partition problem. Annals of Math., 57:531-546, 1953.

[Pfa95] J.L. Pfaltz. Partitions of $2^{n}$. Congressus Numerantium, 109:3-12, 1995.

[Rod69] Öystein Rodseth. Some arithmetical properties of $m$-ary partitions. Proc. Camb. Phil. Soc, 68:447-453, 1969.

[Slo73] N.J.A. Sloane. A Handbook of Integer Sequences. Academic Press, 1973. On-line version at http://www.research.att.com/⒎ Enjas/.

[Tan18a] A. Tanturri. Atti R. Acad. Sci. Torino, 54:69-82, 1918.

[Tan18b] A. Tanturri. Atti R. Acad. Lincei, 27:399-403, 1918. 


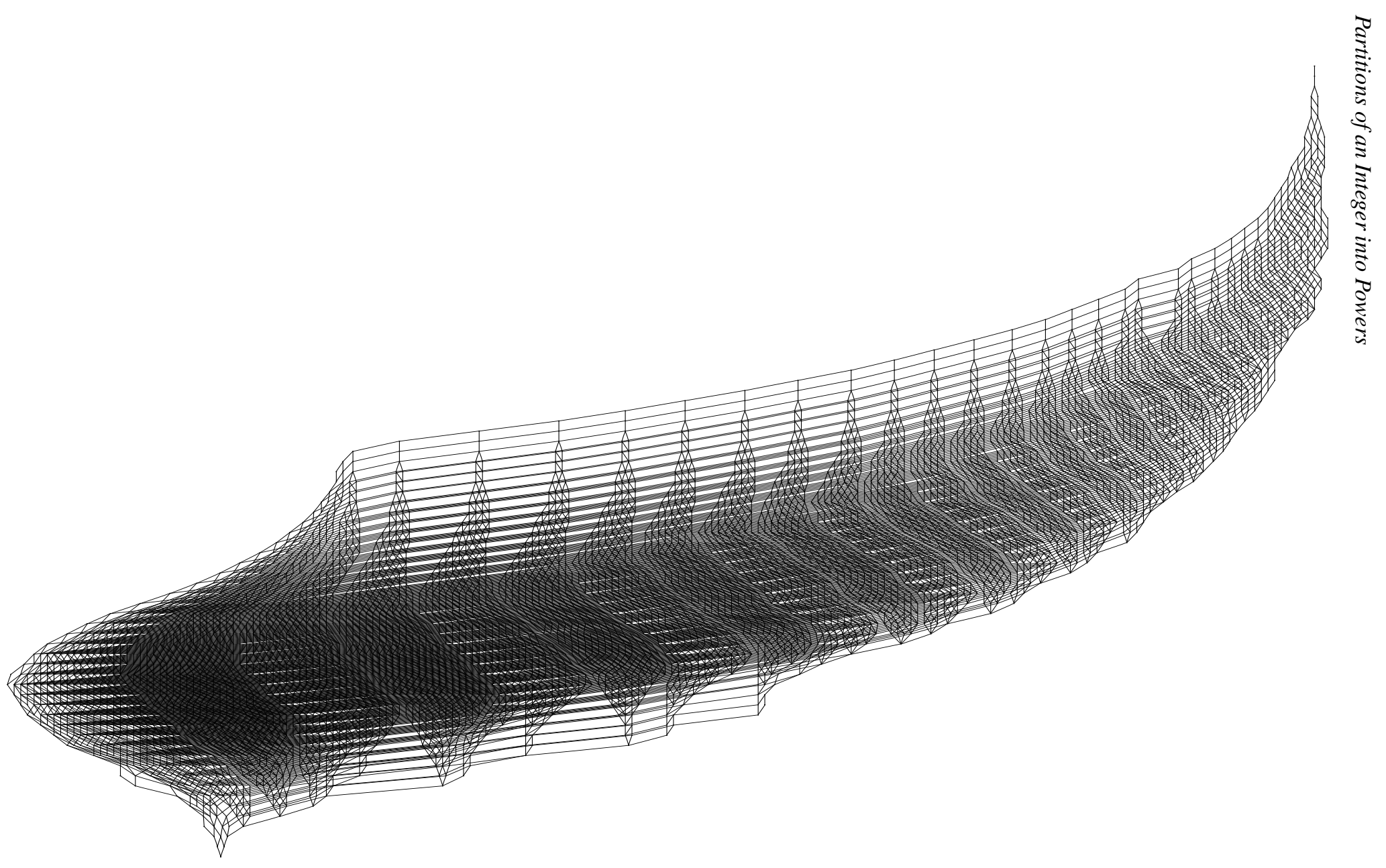

Fig. 4: The distributive lattice $R_{2}(80)$, which contains 4124 elements and 12484 edges. The self-similarity of the set clearly appears on this diagram. 
